\title{
Improved Method for In Vitro Secondary Amastigogenesis of Trypanosoma cruzi: Morphometrical and Molecular Analysis of Intermediate Developmental Forms
}

\author{
L. A. Hernández-Osorio, ${ }^{1,2}$ C. Márquez-Dueñas, ${ }^{1}$ L. E. Florencio-Martínez, ${ }^{1,3}$ \\ G. Ballesteros-Rodea, ${ }^{1,4}$ S. Martínez-Calvillo, ${ }^{3}$ and R. G. Manning-Cela ${ }^{1}$ \\ ${ }^{1}$ Departamento de Biomedicina Molecular, Centro de Investigación y de Estudios Avanzados del IPN, \\ AV. IPN 2508, Col San Pedro Zacatenco, 07360 México, DF, Mexico \\ ${ }^{2}$ Facultad de Medicina y Cirugía, Universidad Autónoma Benito Juárez de Oaxaca, 68020 Oaxaca, Mexico \\ ${ }^{3}$ FES Iztacala, UBIMED, UNAM, 54090 Edo. de México, Mexico \\ ${ }^{4}$ Facultad de Medicina Veterinaria y Zootecnia, UNAM, 04510 México, DF, Mexico \\ Correspondence should be addressed to R. G. Manning-Cela, rmanning@cinvestav.mx
}

Received 10 June 2009; Revised 10 September 2009; Accepted 21 September 2009

Academic Editor: Luis I. Terrazas

Copyright ( 2010 L. A. Hernández-Osorio et al. This is an open access article distributed under the Creative Commons Attribution License, which permits unrestricted use, distribution, and reproduction in any medium, provided the original work is properly cited.

\begin{abstract}
Trypanosoma cruzi undergoes a biphasic life cycle that consists of four alternate developmental stages. In vitro conditions to obtain a synchronic transformation and efficient rates of pure intermediate forms (IFs), which are indispensable for further biochemical, biological, and molecular studies, have not been reported. In the present study, we established an improved method to obtain IFs from secondary amastigogenesis. During the transformation kinetics, we observed progressive decreases in the size of the parasite body, undulating membrane and flagellum that were concomitant with nucleus remodeling and kinetoplast displacement. In addition, a gradual reduction in parasite movement and acquisition of the amastigote-specific Ssp4 antigen were observed. Therefore, our results showed that the in vitro conditions used obtained large quantities of highly synchronous and pure IFs that were clearly distinguished by morphometrical and molecular analyses. Obtaining these IFs represents the first step towards an understanding of the molecular mechanisms involved in amastigogenesis.
\end{abstract}

\section{Introduction}

Trypanosoma cruzi is an obligate intracellular parasite that is responsible for Chagas disease, which affects 16-18 million people in Latin America. This parasite has a complex biphasic life cycle in which four developmental forms alternate between the Reduviid beetle vector (epimastigotes and metacyclic trypomastigotes) and the mammalian host (amastigotes and bloodstream trypomastigotes). Transmission is initiated in the Reduviid beetle vector, which becomes infected by taking up circulating trypomastigotes during a blood meal. After trypomastigotes differentiate to epimastigotes in the insect gut lumen, the parasite divides by binary fission before migrating along the hindgut and rectum, where they transform to metacyclic trypomastigotes.
These trypomastigotes are released near the bite wound with the insect feces during the next blood meal. Following its introduction into mammalian blood, the trypomastigotes penetrate nonphagocytic and phagocytic cells through a parasitophorous vacuole to start the intracellular cycle. In this stage, they differentiate into amastigotes and replicate in the infected cell cytoplasm. Amastigotes develop into nondividing bloodstream trypomastigotes that can either initiate another round of infection to propagate to different organs or can be taken up by the insect vector to complete the life cycle.

Throughout its life cycle, T. cruzi survives under a wide range of environmental conditions that induce complex morphological changes among parasite stages. In addition to the four main developmental forms, it is possible to 
observe intermediate forms (IFs) that seem to follow the same differentiation path, independent of whether they exist in a vertebrate or in an invertebrate host $[1,2]$. Intermediate forms appear transiently during the differentiation of epimastigotes into metacyclic trypomastigotes (metacyclogenesis) in the triatomine, the differentiation of metacyclic trypomastigotes (primary amastigogenesis), and tissue-derived trypomastigotes (secondary amastigogenesis) into amastigotes and also into bloodstream trypomastigotes inside the mammalian host cell [2-5].

Adaptation of T. cruzi to diverse environments found in the different hosts undoubtedly induces a complex regulation of gene expression that apparently precedes the morphological changes observed during parasite transformation. Several researchers have studied some of the factors that represent physiological stress for the parasite and have demonstrated that temperature, nutritional conditions, and $\mathrm{pH}$ stimulate morphological differentiation during amastigogenesis [5-8].

The vast majority of the information regarding in vivo and in vitro amastigogenesis comes from data in which tissue-derived trypomastigotes were used because it was possible to obtain higher yields of these parasites $[3,5,8-15]$. Ultra-structural and molecular analyses during the trypomastigote to amastigote transformation have shown a complex and progressive morphological rearrangement of parasite shape and flagellum that has been associated with the differential expression of stagespecific antigens $[3,5,8]$. A comparative morphology study showed that even when metacyclic and bloodstream trypomastigotes share similar biological and morphological properties, primary and secondary amastigogenesis apparently display different developmental processes, which suggests that their intracellular mechanisms are different [5].

Although the basic features of the amastigogenesis transformation process are known, the molecular mechanisms involved are still unidentified. Analysis of the molecules implicated in the detonation and control of the transformation process will increase our knowledge about morphogenesis and gene expression programs that are involved not only in the differentiation between developmental forms but also during parasite transitions from the nonreplicative to the replicative stage. The usefulness of a differentiation system for molecular research in which a population of cells is involved depends first on the synchrony of the transition, second on the availability of easily analyzable markers for monitoring the process, and third on the system efficiency for obtaining large enough amounts of analyzable sample for further biochemical, biological, and molecular analyses. To date, in vitro conditions that allow for obtaining IFs that satisfy these needs have not been reported. Therefore, in vitro conditions that induce high rates of pure IFs during the transformation of culturederived trypomastigotes into amastigotes are described in this work. Furthermore, morphological, cellular, and molecular characterizations of the different IFs obtained are presented.

\section{Materials and Methods}

2.1. Cells and Parasites. NIH 3T3 fibroblasts were grown in high glucose Dulbecco's minimal essential medium (hgDMEM) supplemented with $10 \%$ fetal bovine serum (FBS), $1 \%$ glutamine, and $5 \mu \mathrm{g} / \mathrm{mL}$ penicillin-streptomycin at $37^{\circ} \mathrm{C}$ in a humidified atmosphere with $5 \% \mathrm{CO}_{2}$ in a $75 \mathrm{~cm}^{2}$ Corning cell culture flask (catalog number 4306-41). Epimastigotes of T. cruzi CL-Brener strain were grown in liver infusion tryptose medium (LYT) containing 10\% FBS and Hemin $(25 \mu \mathrm{g} / \mathrm{mL})$ at $28^{\circ} \mathrm{C}[16]$.

Fifty percent confluent fibroblasts were infected with $2 \times 10^{6}$ CL-Brener mid-log-phase epimastigotes per $\mathrm{mL}$ of hgDMEM $(15 \mathrm{~mL})$ supplemented with $2 \%$ FBS to get a parasite-host cell ratio of $10: 1$. The NIH $3 \mathrm{~T} 3$ monolayers were washed every 2 days with hgDMEM medium until nonadherent parasites were removed, and then fresh hgDMEM plus $2 \%$ FBS was added. The parasites released from the early infections corresponding to the first 6 days after the first liberation were harvested every day at $2000 \times \mathrm{g}$ for 5 minutes. The trypomastigotes were separated from amastigotes by immunoprecipitation using $500 \mu \mathrm{L}$ of hybridoma supernatant of monoclonal antibody $2 \mathrm{C} 2 \mathrm{~B} 6$, which is specific for the Ssp4 surface antigen of amastigotes [3], per $3 \times$ $10^{8}$ parasites at $37^{\circ} \mathrm{C}$ for 1 hour. The trypomastigotes were separated from amastigotes at $800 \mathrm{rpm}$ for 10 seconds in a microcentrifuge and were washed with 1X PBS as many times as necessary to obtain pure parasites. The amastigotes were found in the pellet, and the trypomastigotes were found in the supernatant.

2.2. In Vitro Amastigogenesis. Purified tissue culture-derived trypomastigotes $\left(5 \times 10^{6}\right)$ were harvested at $500 \times \mathrm{g}$ for 10 minutes and were transferred to $100 \times 20 \mathrm{~mm}$ untreated Petri dishes containing $1 \mathrm{~mL}$ per experiment of hgDMEM at different pHs (4, 5, 6, 7, 8, and 9) without or with different FBS concentrations $(0.5 \%, 1 \%, 1.5 \%$, and $2 \%)$ incubated at $37^{\circ} \mathrm{C}$ in a $5 \% \mathrm{CO}_{2}$ atmosphere.

After $1,2,3,4,5,6,12,24,48$, or 72 hours, the parasites were washed, fixed with $4 \%$ paraformaldehyde in 1 XPBS, washed again, and dropped $\left(3 \times 10^{4}\right.$ parasites $/ 10 \mu \mathrm{L}$ ) over poly-L-lysine-treated slides (Silane-Prep slides SIGMA DIAGNOSTICS, catalog number S4651) inside circles delimited with a liquid-repellent slide marker pen (Electron Microscopy Sciences catalog number 71310). After the slides were air dried, the parasites were stained with Hema 3 according to the protocol supplied by the manufacturer (Fisher Diagnostic 840021), and the percentages of trypomastigotes, Ifs, and amastigotes were determined for 100 cells analyzed randomly under an optic microscope.

2.3. Resistance to Complement-Mediated Lysis Assays. Trypomastigotes, amastigotes, epimastigotes, or IFs $\left(2 \times 10^{6}\right.$ parasites) were incubated with $500 \mu \mathrm{L}$ of fresh human serum at $37^{\circ} \mathrm{C}$ for 30,60 , and 120 minutes. Parasite lysis was determined by adding one volume of trypan blue (0.4\% in 1XPBS, Hycel 23850) and counting the complement-resistant parasites in a hemocytometer 
under a light microscope. Three independent experiments, repeated in triplicate each time, were carried out using serum from different donors.

2.4. Localization of Ssp4 by Indirect Immunofluorescence. The parasites were harvested, washed, fixed, and dropped over poly-L-lysine-treated slides as described above. The parasites were permeabilized with $0.1 \%$ Triton-X100 for 5 minutes, rinsed extensively with $1 \mathrm{XPBS}$, and treated with $2 \%$ BSA (Albumin, bovine, Cohn fraction V / $1 \mathrm{X} \mathrm{PBS} \mathrm{pH} \mathrm{7)} \mathrm{at} 37^{\circ} \mathrm{C}$ for 30 minutes. These preparations were washed with PBS and incubated 1 hour at $37^{\circ} \mathrm{C}$ with hybridoma supernatant $(1$ : 50 ) of the amastigote-specific monoclonal antibody $2 \mathrm{C} 2 \mathrm{~B} 6$ [3]. After rinsing, a secondary antimouse antibody tagged with Alexa Fluor 488 green diluted $1: 1000$ was layered on the parasites and incubated for 1 hour at $37^{\circ} \mathrm{C}$. The slides were rinsed, mounted with Vectashield Mounting Medium (Vector Laboratories Cat. H-1000), and observed under an Olympus fluorescence microscope (BX41) equipped with a 60X/1.25 Oil Iris Ph3 UPlanFL N objective. The images were captured using an Evolution VF Fast Cooled Color Media Cybernetics camera and were analyzed using the Image-Pro Plus V 6.0 Media Cybernetics program.

2.5. DAPI Staining. The parasites were processed and analyzed as per the indirect immunofluorescence protocol described above, with some specifications. After treatment with a polyclonal antibody against the epimastigotes $(1: 200)$ obtained from the mouse and the antimouse antibody tagged with Alexa Fluor 546 red $(1: 1000)$ as the first and second antibodies, the parasites were stained with $10 \mu \mathrm{g} / \mathrm{mL}$ DAPI (Molecular Probes). The slides were mounted and analyzed as described above.

\section{Results}

3.1. Serum-Free Culture at pH5 Stimulated the Highest Rates of Transformation from Tissue-Derived Trypomastigotes into Amastigotes. As mentioned before, conditions such as temperature, nutritional environment, and $\mathrm{pH}$ stimulate the in vitro differentiation of trypomastigotes into amastigotes [58 ]. To determine the best conditions for obtaining the highest rates of transformation, in vitro experiments were performed using tissue-derived trypomastigotes incubated at $37^{\circ} \mathrm{C}$ in hgDMEM medium at different pHs $(4,5,6,7,8$, and 9), without or with $0.5 \%, 1 \%, 1.5 \%$, or $2 \%$ FBS for different lengths of time, as described in the materials and methods. The movement and morphology of the parasites were analyzed in fresh preparations under a light microscope, and the relative percentages of trypomastigotes, Ifs, and amastigotes were determined. In all of the conditions in which parasite differentiation was achieved, the trypomastigotes showed gradual morphological changes and size reduction; they transformed from the typical S- or C-shaped bloodstream morphology to diverse IFs that finally reached the rounded or oval shape characteristic of amastigotes after 12 hours of incubation. A progressive reduction of parasite movement concomitant with the gradual shortening of the undulating membrane and flagellum was also observed.

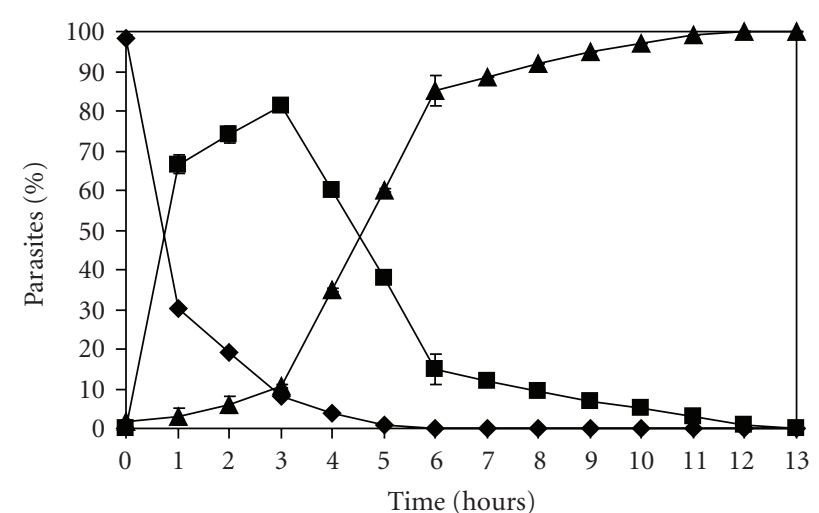

Figure 1: Transformation kinetics from tissue-derived trypomastigote to amastigote in hgDMEM at $\mathrm{pH} 5$ without FBS at $37^{\circ} \mathrm{C}$ : relative percentage of trypomastigotes (- - ), IFs (-ם-), and amastigotes $\left(-\boldsymbol{\Delta}_{-}\right)$analyzed in fresh preparations under the light microscope. The results are the average of 3 independent experiments.

Although similar morphological patterns of differentiation could be seen in some of the different conditions tested, very different rates of transformation were obtained. Incubation at $37^{\circ} \mathrm{C}$ in hgDMEM at $\mathrm{pH} 5$ without $\mathrm{FBS}$ induced the most efficient rates of differentiation. Under these conditions, the level of tissue-derived trypomastigotes decreased concomitantly with increasing amounts of IFs, which reached $85 \%$ within 3 hours of incubation (Figure 1). Subsequently, as the percentage of IFs decreased, the relative amount of amastigotes increased, and the transformation process was completed after 12 hours of incubation. A homogenous population (100\%) of round-shaped forms, which displayed either no flagella or very short protruding flagella and a noticeable reduction of movement, was observed at this point. On the contrary, when tissuederived trypomastigotes were incubated in hgDMEM at $\mathrm{pH}$ 4 without FBS, only $30 \%$ of the cells were transformed into amastigotes after 3 hours and $65 \%$ after 12 hours (data not shown). In addition, only $21 \%$ of the trypomastigotes differentiated into amastigotes after 24 hours of incubation at pH 6 without FBS (data not shown). Finally, at pHs 7, 8, and 9 in the absence of FBS as well as those same pHs with $0.5 \%$, $1 \%, 1.5 \%$, and $2 \%$ FBS did not induce parasite differentiation (data not shown).

These results indicate that the differentiation process during the in vitro secondary amastigogenesis involves several IFs as a result of the morphological change of the parasites. In addition, the optimal conditions to induce the highest rates of transformation from tissue-derived trypomastigotes into amastigotes were $\mathrm{pH} 5$, serum-deprivation and $37^{\circ} \mathrm{C}$.

\subsection{Gradual Morphological Changes were Observed During In} Vitro Secondary Amastigogenesis. The different developmental forms of T. cruzi can be identified by morphological and biological parasite features, such as the size and shape of the parasite, undulating membrane and flagellum, the position 
of flagellum emergence, and the size, shape, and relative position of the nucleus and the kinetoplast. The amastigote has a spherical- to oval-shaped form that is 2 to $4 \mu \mathrm{m}$ in diameter with no or very short free flagellum and a kinetoplast located in the middle of the parasite body close to the nucleus. The trypomastigote kinetoplast is located subterminal and posterior to the nucleus. The trypomastigotes have a flagellum and an undulating membrane that extends the entire length of the parasite $(16-25 \mu \mathrm{m}$ long and $2 \mu \mathrm{m}$ diameter). Trypomastigotes found in the blood of infected mammals have a slender S- or C-shaped form, while the trypomastigotes found in infected insects usually have a straight form [17].

Once the best conditions to induce the transformation of the parasite were determined, a detailed morphological analysis was carried out on fixed and stained preparations, and the relative amounts of trypomastigotes, amastigotes, and IFs were determined at different points of the differentiation kinetics at $37^{\circ} \mathrm{C}$ using hgDMEM at $\mathrm{pH} 5$ with serumdeprivation (Figure 2). This methodology allowed us to perform a morphometrical analysis and a more precise quantification of the transformation process compared to those obtained with fresh preparations. Before initiating the differentiation process, tissue-derived trypomastigotes showed the typical morphology previously described (Figure 2a). During the transformation process, the parasite progressively reduced its entire length by $22 \%$ at 1 hour, $23 \%$ at 2 hours, $39 \%$ at 3 hours, $48 \%$ at 4 hours, $50 \%$ at 5 hours, and $53 \%$ at 6 hours of transformation as shown in Figure 2 and Table 1. The quantitative analysis indicated that as little as 1 hour of transformation time was enough to induce high rates of IFs (80\%) in which the parasites displayed a widening and shortening of their body, flagellum, and undulating membrane; however, this last one maintained the subterminal emergence site that is characteristic of trypomastigotes (Figure $2 \mathrm{~b}$ and Table 1). After 2 hours of transformation, no trypomastigotes were detected, which indicates that the culture contained $100 \%$ IFs. These IFs showed size reduction, a light oval-shaped form, shortening of their flagellum, and an undulating membrane in which their emergence site was slightly displaced to the middle of the parasite body (Figure 2(c) and Table 1). After 3 hours, the morphology of the IFs was very close to the one observed at 2 hours of transformation, but their size continued to reduce and the undulating membrane was not appreciable (Figure 2(d) and Table 1). After 4 and 5 hours of transformation, the IFs showed similar size and morphology to the amastigotes and were only distinguished by the progressive shortening of their flagellum (Figures 2(e) and 2(f) and Table 1). Finally, after 6 hours of transformation, the parasites reached the sphericalto oval-shaped form with no or very short free flagellum (Figure 2(g)), and their morphology was indistinguishable from the one observed in the cell culture-derived amastigotes (Figure 2(h) and Table 1). To assess the reversibility of the differentiation process, the IFs were cultured again in hgDMEM at pH 7.2 complemented with $2 \%$ FBS after 1, 2, $3,4,5,6,12,24,48$ or 72 hours of differentiation at $37^{\circ} \mathrm{C}$ using hgDMEM at $\mathrm{pH} 5$ with serum deprivation. In all cases, the IFs continued their differentiation, thus indicating that

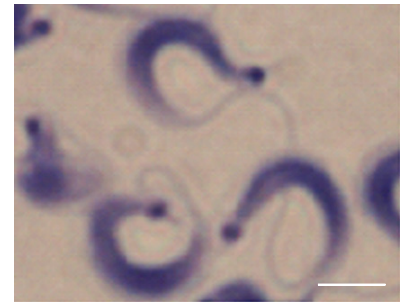

(a)

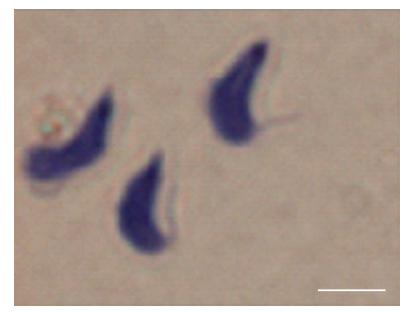

(b)

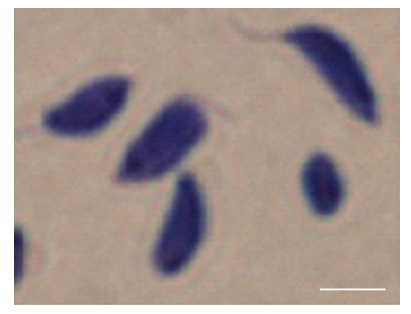

(c)

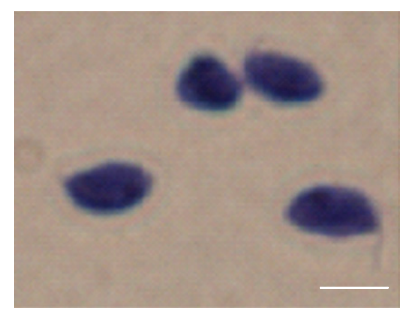

(d)

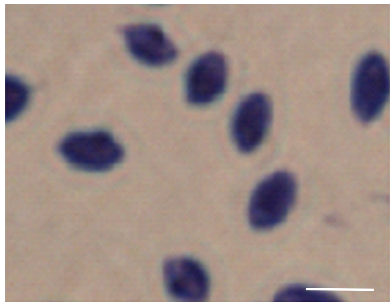

(h)

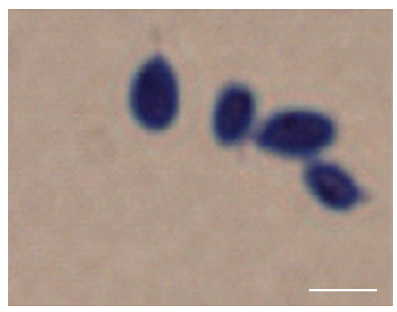

(g)

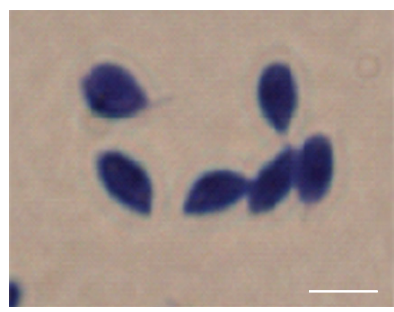

(f)

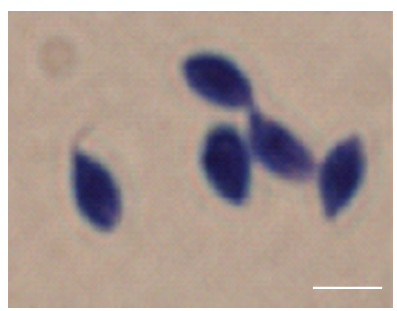

(e)
FIGURE 2: Morphological analysis of the kinetics of extracellular differentiation from tissue-derived trypomastigotes (a) to amastigotes (h), in hgDMEM at $\mathrm{pH} 5$ without $\mathrm{FBS}$ at $37^{\circ} \mathrm{C}$ : intermediate forms at 1 hour (b), 2 hours (c), 3 hours (d), 4 hours (e), 5 hours (f), and 6 hours (g) of transformation stained with Hema 3 . Bar $=25 \mu \mathrm{m}$.

once detonated, this process cannot be stopped and is not reversible (data not shown).

These results showed that, under the in vitro conditions used, the trypomastigotes differentiated very efficiently into highly homogeneous and pure IF populations after 2 hours of induction.

\subsection{The Shape and Position of the Nucleus and Kinetoplast} Determined the Different IFs. To continue with the morphological analysis of the IFs, the shape and position of the nucleus and the kinetoplast at each point of the transformation kinetics were determined. The parasites were then 
stained with a polyclonal antibody against epimastigote total proteins, which had been previously shown to recognize all of the developmental forms and IFs of T. cruzi. The reactions were developed with an Alexa Fluor 546 red-conjugated antimouse antibody, and the kinetoplast and nucleus were counterstained with DAPI.

As shown in previous experiments, the same morphological transformation pattern of the parasite body was found with this technique (compare Figures 2 and 3). Nucleus remodeling and a progressive and continuous displacement of the kinetoplast from the posterior to the anterior position with respect to the parasite nucleus were also observed. As shown in Table 1, the distance between the nucleus and the kinetoplast became shorter during the transformation kinetics. At 1 hour of transformation (Figure 3(b)), the distance between both organelles was reduced by $28 \%$ (Table 1), even though the IFs showed a subterminal kinetoplast posterior to the nucleus, as commonly observed in trypomastigotes (Figure 3(a)). After 2 hours of transformation, the IFs showed a displacement of their kinetoplast to the middle of the parasite body and reduced the distance with respect to the nucleus (now remodeled from the lengthened-shaped form to an oval-shaped form) by $58 \%$ (Figure 3(c) and Table 1). After 3 hours of transformation, the kinetoplast remained located posterior to the nucleus, close to the middle part of the parasite body, with a $66 \%$ reduction in the distance between both organelles (Figure 3(d) and Table 1). At 4 hours of transformation, the kinetoplast was positioned next to and parallel to the nucleus in a transversal position in the middle of the parasite body and showed $85 \%$ reduction in the organelle distance (Figure 3(e) and Table 1). After 5 hours of transformation, the kinetoplast moved to an anterior position with respect to the nucleus, in the middle part of the parasite body and showed a $90 \%$ reduction in the distance between the organelles (Figure 3(f) and Table 1). Finally, at 6 hours of transformation (Figure $3(\mathrm{~g})$ and Table 1 ), the parasite morphology and the position of the kinetoplast were very similar to those observed in the tissue-derived amastigotes (Figure $3(\mathrm{~h})$ and Table 1 ).

These results showed that, concomitant with the morphological parasite shape transformation, nucleus remodeling and kinetoplast displacement are observed during the transformation kinetics under the in vitro conditions used.

\subsection{Highly Synchronous Morphologic Changes were Obtained} During in Vitro Transformation. The morphological analysis described above showed homogenous IF populations; nevertheless, the nucleus remodeling and kinetoplast displacement studies indicated that each point of the transformation kinetics is composed of 3 different IFs. The percentages of trypomastigotes, amastigotes and the different IFs were then determined for each time point evaluated in the previous experiment. As shown in Figure 4, 3 different IFs were observed at 1, 2, 3, 4 and 5 hours of in vitro differentiation (Figures 4(a) and 4(c)). At 1 hour of transformation The first IF, which was arbitrarily named IF1, showed similar morphology to the immediate previous parasite stage (trypomastigotes for the first hour and IF3 for 2, 3, 4, and 5 hours)

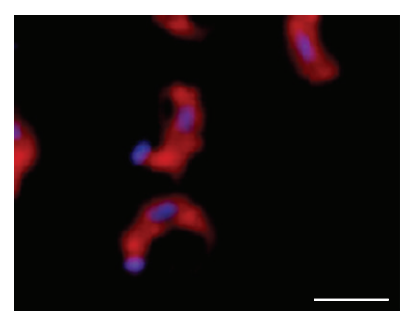

(a)

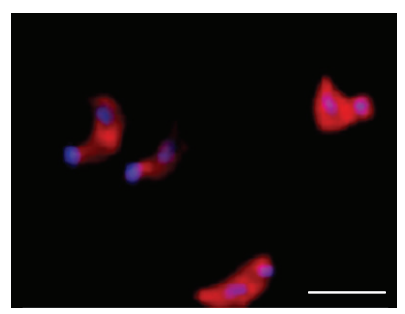

(b)

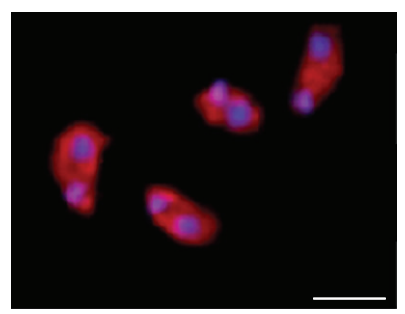

(c)

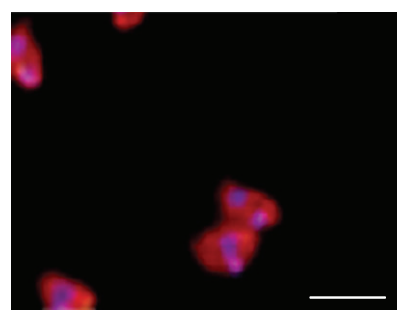

(d)

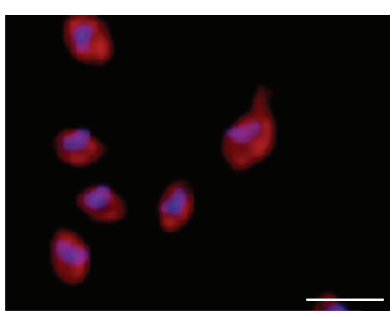

(h)

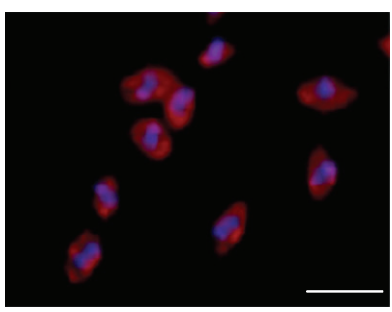

(g)

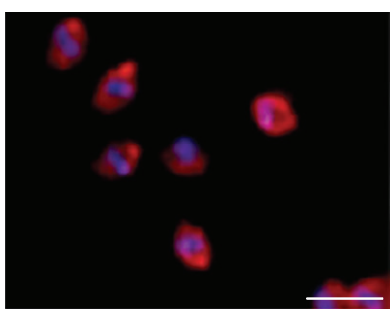

(f)

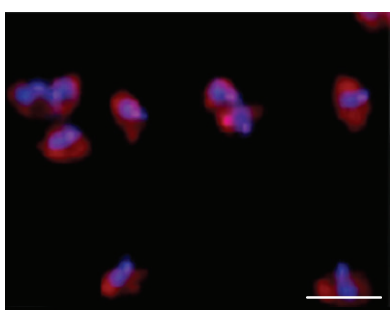

(e)
FigURE 3: The shape and position of the nucleus and the kinetoplast identified the different IFs. The morphology and position of the nucleus and the kinetoplast were determined by indirect immunofluorescence and DAPI staining of trypomastigotes (a), IFs at 1 hour (b), 2 hours (c), 3 hours (d), 4 hours (e), 5 hours (f) and 6 hours (g) of transformation and amastigotes (h). Bar $=25 \mu \mathrm{m}$.

and represented $2 \%$ to $3 \%$ of the total parasites. The second intermediary form, named IF2, showed a slightly, but clearly, more differentiated morphology than IF1 and represented the most abundant parasite population ( $85 \%$ to $90 \%)$. The third IF, still more differentiated than IF2 and named IF3 ( $2 \%$ to $15 \%$ ), displayed characteristics similar to the IF1 that emerged from the next time of transformation. At 1 hour of transformation, trypomastigotes comprised only $2 \%$ of the culture, which confirms that the transformation process is very efficient under the established conditions (Figures 4(a), 4(b) and 4(c)). Moreover, from 1 to 5 hours of transformation were not observed any amastigotes (Panel A). 
TABLe 1: Morphometrical analysis of T. cruzi developmental stages.

\begin{tabular}{lcc}
\hline Parasite & Parasite length $(\mu \mathrm{m})$ & $\begin{array}{c}\text { Distance between } \\
\text { nucleus and } \\
\text { kinetoplast }(\mu \mathrm{m})\end{array}$ \\
\hline Trypomastigote & $24.17 \pm 4.06$ & $15.19 \pm 3.02$ \\
If 1 hours & $19.04 \pm 2.62$ & $10.89 \pm 1.97$ \\
If 2 hours & $18.52 \pm 2.43$ & $6.43 \pm 1.60$ \\
If 3 hours & $14.84 \pm 2.47$ & $5.12 \pm 1.58$ \\
If 4 hours & $12.52 \pm 1.03$ & $2.29 \pm 0.72$ \\
If 5 hours & $11.98 \pm 1.44$ & $1.47 \pm 0.53$ \\
If 6 hours & $11.26 \pm 1.24$ & $1.36 \pm 0.38$ \\
Amastigote & $11.45 \pm 1.02$ & $1.08 \pm 0.22$ \\
\hline
\end{tabular}

The results are the average of 100 cells analyzed randomly.

Finally, at 6 hours of transformation, $100 \%$ of the parasites showed a very similar morphology to that of tissue-derived amastigotes (Figures $3(\mathrm{~g})$ and $3(\mathrm{~h})$, Table 1 and Figures $4(\mathrm{a})$, $4(\mathrm{~b})$ and $4(\mathrm{c})$ ).

The only forms detected after 2, 3, 4 and 5 hours of transformation were IFs with a gradually increasing degree of differentiation. Therefore, this experimental model could be useful for future analysis of the differential expression between the IFs and the completely differentiated forms of T. cruzi. In addition, even when the IFs at 1 hour of transformation showed morphometric characteristics that corresponded with an IF, the presence of contaminant trypomastigotes $(2 \%)$ rendered this population not useful for differential expression studies (Figure 4).

\subsection{Secondary Amastigogenesis was Accompanied by the} Gradual Acquisition of the Amastigote-Specific Ssp4 Glycoprotein. The previous results demonstrated that different IFs, which can be identified by specific morphological characteristics, are generated during amastigogenesis. Since it was necessary to know more about their biological properties, a molecular marker was evaluated for the different IFs during the transformation kinetics. It has been reported that the morphological differentiation of the IFs implies the remodeling of parasite surface molecules $[3,8]$. The expression of the amastigote-specific surface glycoprotein Ssp4 was evaluated by indirect immunofluorescence in the diverse IFs as well as in trypomastigotes and amastigotes used as controls.

The results presented in Figure 5 showed that, as expected, the trypomastigotes did not express the amastigote-specific surface glycoprotein (Figures 5(a) and 5(b)), while amastigotes displayed heterogeneous levels of Ssp4 (Figures 5(o) and 5(p)). Moreover, while the IFs generated at 1,2 , and, 3 hours of transformation did not express Ssp4 (from Figures 5(c) to 5(h)), approximately $8 \%$ and $36 \%$ of parasites at 4 (Figures 5(i) and $5(\mathrm{j})$ ) and 5 hours of differentiation (Figures $5(\mathrm{k})$ and $5(\mathrm{l})$ ), respectively, showed low expression levels. In addition, after 6 hours of transformation (Figures $5(\mathrm{~m})$ and $5(\mathrm{n})$ ), approximately $76 \%$ of the parasites showed variable and lower levels of Ssp4 compared to culture-derived amastigotes (Figures 5(o) and

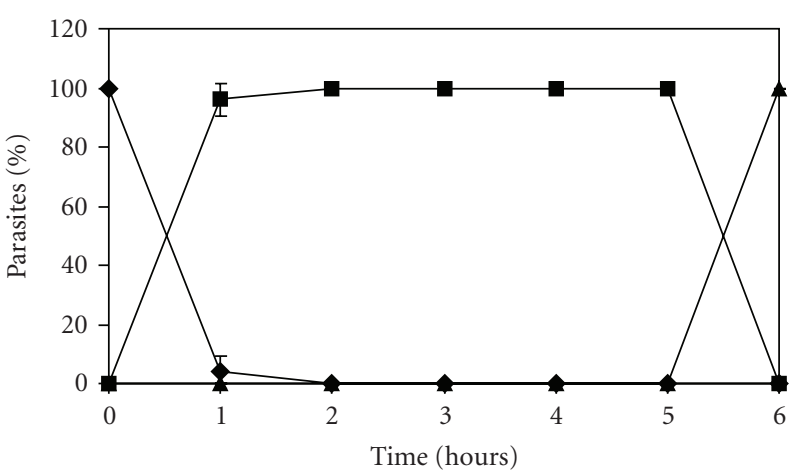

(a)

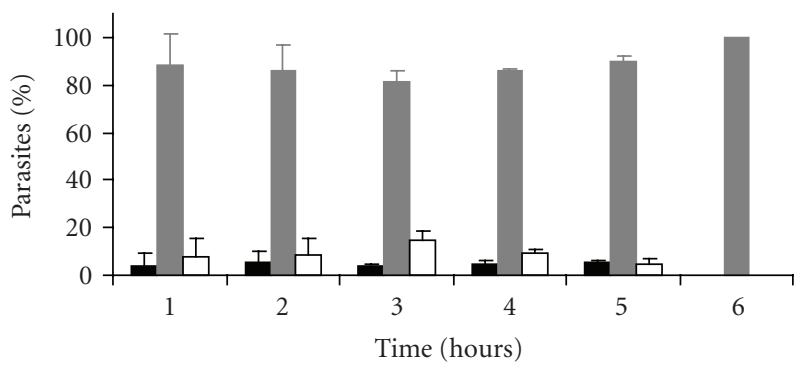

(b)

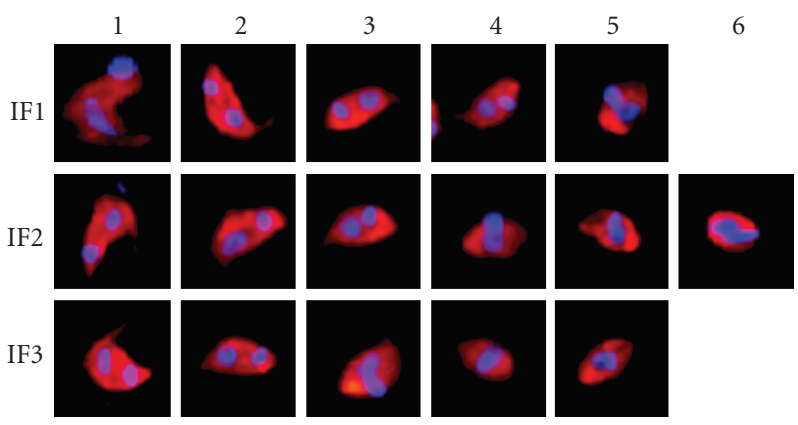

(c)

FIGURE 4: Highly synchronous morphologic changes are obtained during the in vitro transformation kinetics. Quantitative analysis of 2 independent experiments of extracellular differentiation from tissue-derived trypomastigotes into amastigotes stained by indirect immunofluorescence and DAPI after 1, 2, 3, 4, 5 and 6 hours of transformation. (a) Relative percentage of trypomastigotes (- - ), IFs (-口-) and amastigotes (- $\mathbf{-}-)$. (b) Three different IFs arbitrarily designated as IF1 (black), IF2 (grey) and IF3 (white) were observed from 1 to 5 hours of transformation. (c) Representative parasite of the corresponding IFs of panel $b$.

5(p)). Finally, after 72 hours of transformation, all parasites showed equivalent expression of Ssp4 to that of culturederived amastigotes (data not show).

These results indicated that the consecutive and gradual expression of Ssp4 was concomitant with the morphological transformation of trypomastigotes into amastigotes, in which the IFs with a similar morphology to amastigotes were the ones that started expressing the amastigote-specific glycoprotein. Moreover, even though the morphology of 


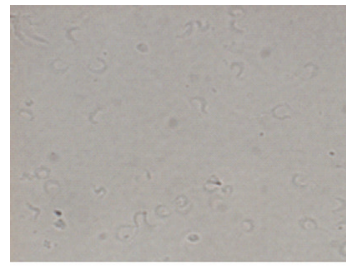

(a)

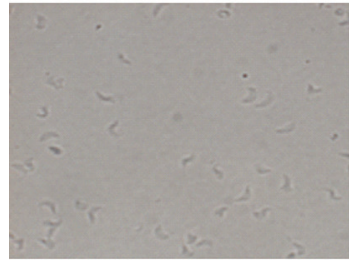

(c)

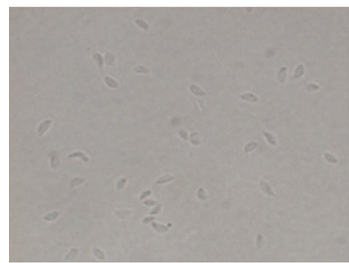

(e)

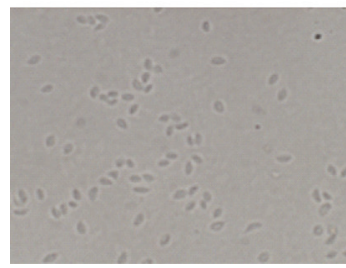

(g)

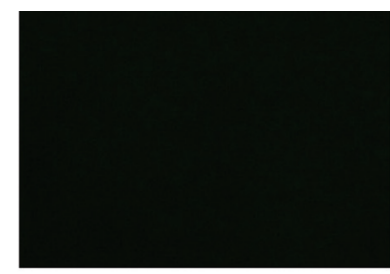

(b)

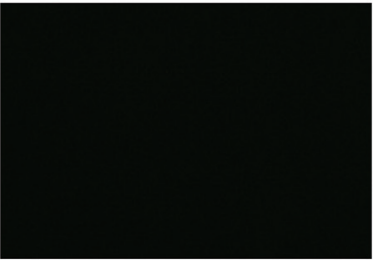

(d)

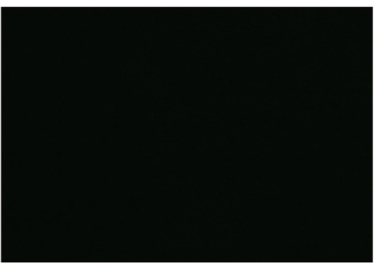

(f)

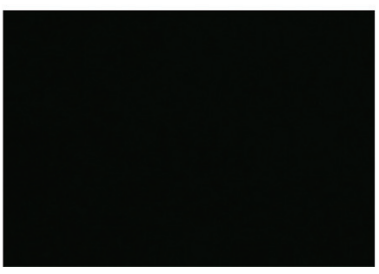

(h)

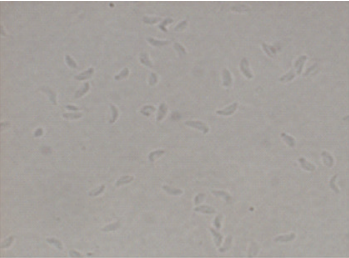

(i)

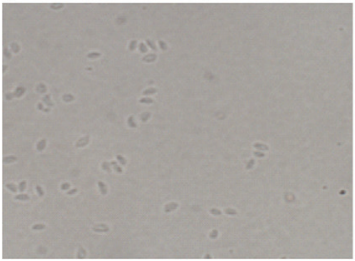

(k)

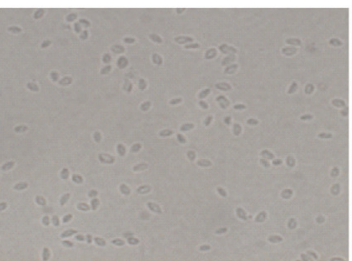

$(\mathrm{m})$

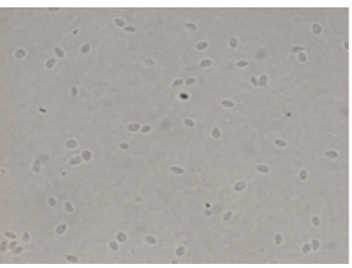

(o)

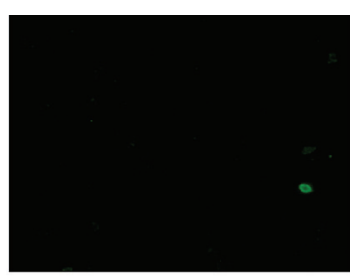

(j)

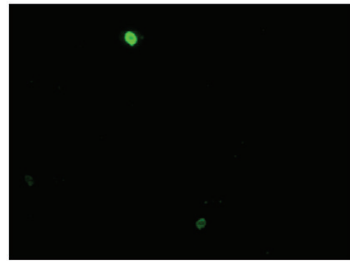

(1)

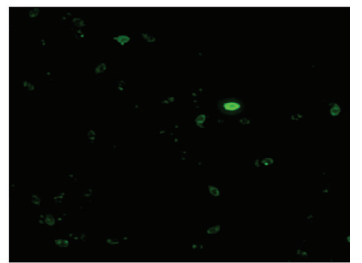

(n)

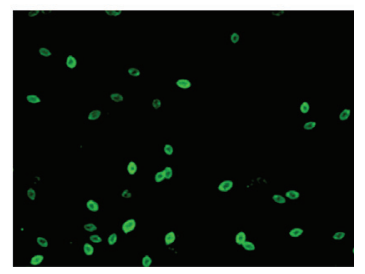

(p)

FIgURE 5: Secondary amastigogenesis is accompanied by the gradual acquisition of amastigote-specific Ssp4 surface glycoprotein. The localization of Ssp4 was determined by indirect immunofluorescence in trypomastigotes (a, b), IFs at 1 hour (c, d), 2 hours (e, f), 3 hours (g, h), 4 hours (i, j), 5 hours $(\mathrm{k}, \mathrm{l})$ and 6 hours $(\mathrm{m}, \mathrm{n})$ of transformation and in amastigotes $(\mathrm{o}, \mathrm{p})$. The right panels are the immunofluorescence image (b, d, f, h, j, l, n, p) of the corresponding light microscopy panels (a, c, e, g, i, k, m, o).

the parasites at 6 hours of transformation was similar to the tissue-derived amastigote morphology, the expression of this molecular marker indicated that these parasites were not completely transformed at the molecular level.

3.6. The IFs were Resistant to Complement-Mediated Lysis. Continuing with the biological analysis of the different IFs, their resistance to complement-mediated lysis was determined. As the IFs are obtained during trypomastigote differentiation into amastigotes and both developmental stages are resistant to complement-mediated lysis, it would not be surprising if the IFs are also resistant. However, it was important to evaluate this phenotype because (1) the IFs are usually intracellular and therefore not exposed to the blood and extracellular space in the vertebrate host and so they would not need to be resistant to complement-mediated lysis and (2) the IFs obtained during metacyclogenesis and amastigogenesis show very similar morphological characteristics, and very little is known about biological properties that could be used to distinguish them. The IFs obtained by metacyclogenesis are sensitive to complement-mediated lysis [18]. However, this biological property has not been evaluated in the IFs obtained during amastigogenesis. Therefore, the complement-sensitivity of the IFs obtained was evaluated using fresh human serum as described in the materials and methods.

As shown in Figure 6, the IF at 3 hours of transformation, similar to trypomastigotes and amastigotes, was resistant to complement-mediated lysis. Moreover, all of the IFs obtained through the transformation kinetics were also resistant to complement-mediated lysis (data not shown). As expected, epimastigotes were sensitive to complement, thus reaching $100 \%$ lysed parasites in the first 30 minutes of treatment.

These results suggest that the IFs obtained during the in vitro amastigogenesis do not lose the ability to resist 


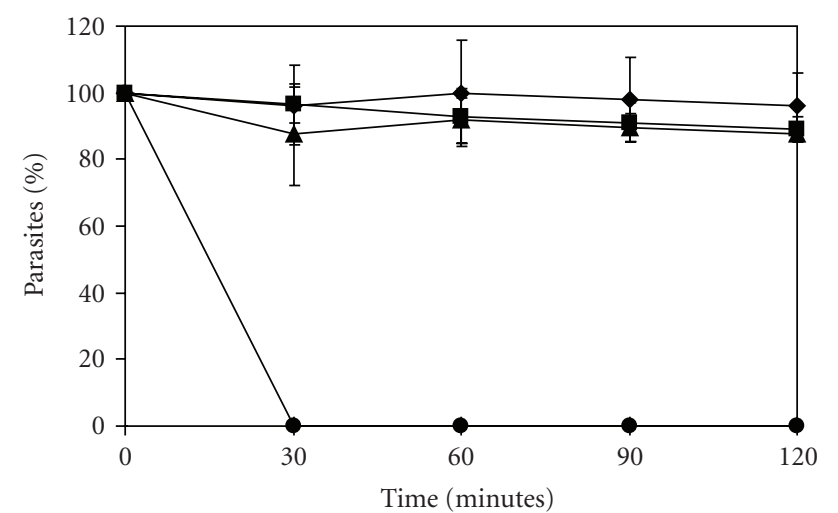

FIGURE 6: Analysis of resistance to complement-mediated lysis. The relative percentages of trypomastigotes (- - -), amastigotes (- - -), IFs at 3 hours of transformation $\left(-\boldsymbol{\Delta}^{-}\right)$and epimastigotes $(-\bullet-)$ were determined after treatment with fresh human serum. The results are the average of 3 independent experiments.

complement-mediated lysis. Therefore, even if the IFs from metacyclogenesis and amastigogenesis are morphologically similar, they have different biological properties that can be used to distinguish them.

\section{Discussion}

The lack of effective in vitro conditions to obtain high rates of synchronic and pure IFs has delayed the understanding of the molecular mechanisms that mediate the cellular remodeling and the identification of molecular markers that are important for amastigogenesis. In the present work, we established an improved method to obtain high rates of pure IFs and correlated parasite differentiation with the expression of a specific developmental marker, complementmediated lysis sensitivity and morphometrical analysis of the nucleus, kinetoplast and parasite body throughout the complete transformation process.

The efficient transformation of cell culture-derived trypomastigotes to amastigotes was induced using an acidic $\mathrm{pH}$ and serum deprivation at $37^{\circ} \mathrm{C}$ (Figure 1). The parasites showed dramatic cell restructuring with a gradual and progressive reduction of their size and the readsorption of their flagellum and undulating membrane until they reached the typical round shape of tissue-derived amastigotes (Figure 2). These results are consistent with previous reports that document the existence of several intermediate forms during the life cycle of T. cruzi, either under in vivo or in vitro conditions that showed the same gradual and progressive parasite morphometrical transformation, apparently as an obligatory step for the differentiation process $[1,3,5,8,18$ 22]. The quantitative analysis demonstrated that parasite transformation was initiated immediately after the exposure to hgDMEM at $\mathrm{pH} 5$ and $37^{\circ} \mathrm{C}$, thus reaching $100 \%$ IFs after only 2 hours of incubation (Figure 4); this indicates a faster and more efficient differentiation rate than previously reported $[5,8]$. These differences could be the result of the absence of BSA in the differentiation medium used in this study in comparison with the $0.4 \%$ BSA contained in the previously reported medium $[5,8]$. In addition, the condition of the cell culture-derived trypomastigotes used to initiate parasite differentiation may play an important role in the process. The parasites used in all of the experiments in this study were early released trypomastigotes that were obtained from early infections corresponding to the first 6 days after the first trypomastigote liberation, because the parasites released later showed variable, not synchronous and inefficient transformation rates. Interestingly, Andrews et al [3] , and Tomlinson et al [8], also used only trypomastigotes early released from infected cells for their transformation experiments. Even when these authors did not justify this specification, it was most probably the result of similar observations.

The morphological restructuring of $T$. cruzi during amastigogenesis has shown that the transition from the slender to the spherical or oval parasite shape comprises an extensive remodeling of cellular architecture, the flagellum and the undulating membrane, and a dramatic change in the overall parasite size, apparently as a result of the reorganization of their subpellicular microtubules[17]. This dramatic restructuring also affects the location and morphology of the parasite organelles during T. cruzi differentiation. The kinetoplast of the trypomastigotes is spherical; whereas in amastigotes, it is elongated $[8,21,23,24]$. In contrast, the nuclei of the trypomastigotes are elongated, apparently following the parasite's shape; meanwhile, it is round in amastigotes. These morphometrical characteristics of the nucleus and the kinetoplast have been reported to allow the distinction of the T. cruzi developmental stages [17]. Therefore, this was the criteria used to initiate the characterization of the IFs obtained (Figure 3 and Table 1). The results showed that the morphometrical analysis of the nucleus and the kinetoplast allows the clear identification of the different IFs. The trypomastigotes showed a bigger distance between the nucleus and the kinetoplast than did the amastigotes. In addition, during amastigogenesis, together with the parasite shape change, we observed nucleus remodeling and a progressive and continuous displacement of the kinetoplast from the posterior to the anterior position with respect to the parasite nucleus. Similar observations were reported by Contreras and collaborators in 2002, during primary amastigogenesis. As in the quantitative analysis conducted during the morphometrical characterization of the IFs (Figure 2 and Table 1), the shape and position of the nucleus and the kinetoplast (Figure 3 and Table 1) allowed us to very precisely determine that one of these populations was highly predominant even when three different IF populations were present at each time of the transformation kinetics (from $85 \%$ to $90 \%$ ), which confirmed a high efficiency of the homogeneity and synchronicity of the differentiation process in our in vitro conditions (Figure 4).

Differentiation of the diverse developmental stages of $T$. cruzi has been associated with changes in their biological properties and with the expression of stage-specific surface molecules [9]. Moreover, the morphological transformation during primary amastigogenesis has been associated with changes in the glycoprotein profile related to the expression of trans-sialidase and mucin previously described in $T$. cruzi. The same behavior was observed in this work during 
the secondary amastigogenesis: contrary to the positive expression of Ssp4 observed in the amastigote surface, the trypomastigotes, epimastigotes and IFs at 1,2 and 3 hours of transformation did not express this amastigotespecific glycoprotein (GP) (Figure 5). In the case of the IFs obtained at 4, 5 and 6 hours of transformation, low and heterogeneous levels of Ssp4 expression were observed. At these times, the total number of parasites expressing Ssp4 as well as the expression level of this GP on their surfaces, increased in parallel with the degree of transformation from trypomastigotes to amastigotes, which confirms that the progressive expression of Ssp4 can be useful as a molecular marker of differentiation $[3,8,21]$.

To initiate and establish infection in the mammalian host, T. cruzi needs to develop several strategies to avoid the immune response. To survive and disseminate in the bloodstream and in the extracellular space, trypomastigotes and amastigotes have developed a stage-specific capacity to resist complement-mediated lysis [25-27], apparently using stage-specific regulators [28-30]. Conversely, epimastigotes found in the insect vector activate the complement cascade, but they are not able to inhibit its lytic action [27]. Complement-mediated lysis of the IFs obtained during in vitro secondary amastigogenesis was evaluated. As expected, trypomastigotes and amastigotes, but not epimastigotes, were resistant to the lytic effects of complement. Besides this, the IFs obtained during in vitro amastigogenesis were not sensitive to complement lysis (Figure 6). This result indicates that these IFs express the proteins that participate in the stage-specific inhibition of complement activation. Nevertheless, future studies will be necessary to elucidate which stage-specific proteins from trypomastigotes and/or amastigotes are expressed by the IFs in order to inhibit complement activation. It has been reported that the IFs generated during the transformation from epimastigote to trypomastigote are sensitive to complement lysis [18]. The IFs obtained during the metacyclogenesis and amastigogenesis are morphologically indistinguishable, which is remarkable, because this biological property might be used as a criterion to distinguish both types of IFs. Moreover, this result further supports the existence of the alternative parasite life subcycle that was previously proposed and the hypothesis that this could be the result of the premature lysis of the infected cells or the extracellular parasite differentiation [3].

In summary, we have shown that the experimental differentiation model reported in this work provides, for the first time, a highly synchronous system that could be useful for studying the molecular mechanism of the transformation process and the identification of the regulators involved in differentiation control, with the potential to identify routes to block the cycle of T. cruzi infection.

\section{Acknowledgements}

This work was supported by CONACYT Grants no. 42862 and 60152 awarded to R. G. Manning-Cela. L. A. HernándezOsorio received a master scholarship from CONACYT (México).

\section{References}

[1] M. Almeida-De-Faria, E. Freymuller, W. Colli, and M. J. Alves, "Trypanosoma cruzi: characterization of an intracellular epimastigote-like form," Experimental Parasitology, vol. 92, no. 4, pp. 263-274, 1999.

[2] K. M. Tyler and D. M. Engman, "The life cycle of Trypanosoma cruzi revisited," International Journal for Parasitology, vol. 31, no. 5-6, pp. 472-481, 2001.

[3] N. W. Andrews, K. S. Hong, E. S. Robbins, and V. Nussenzweig, "Stage-specific surface antigens expressed during the morphogenesis of vertebrate forms of Trypanosoma cruzi," Experimental Parasitology, vol. 64, no. 3, pp. 474-484, 1987.

[4] A. R. Avila, B. Dallagiovanna, S. F. Yamada-Ogatta, et al., "Stage-specific gene expression during Trypanosoma cruzi metacyclogenesis," Genetics and Molecular Research, vol. 2, no. 1, pp. 159-168, 2003.

[5] M. C. Navarro, A. R. De Lima, J. Askue, and V. T. Contreras, "Morphological comparison of axenic amastigogenesis of trypomastigotes and metacyclic forms of Trypanosoma cruzi," Memórias do Instituto Oswaldo Cruz, vol. 98, no. 1, pp. 83-91, 2003.

[6] V. T. Contreras, M. C. Navarro, A. R. De Lima, et al., "Production of amastigotes from metacyclic trypomastigotes of Trypanosoma cruzi," Memorias do Instituto Oswaldo Cruz, vol. 97, no. 8, pp. 1213-1220, 2002.

[7] T. A. Minning, J. Bua, G. A. Garcia, R. A. Mcgraw, and R. L. Tarleton, "Microarray profiling of gene expression during trypomastigote to amastigote transition in Trypanosoma cruzi," Molecular and Biochemical Parasitology, vol. 131, no. 1, pp. 5564, 2003.

[8] S. Tomlinson, F. Vandekerckhove, U. Frevert, and V. Nussenzweig, "The induction of Trypanosoma cruzi trypomastigote to amastigote transformation by low pH," Parasitology, vol. 110, part 5, pp. 547-554, 1995.

[9] B. A. Burleigh and N. W. Andrews, "The mechanisms of Trypanosoma cruzi invasion of mammalian cells," Annual Review of Microbiology, vol. 49, pp. 175-200, 1995.

[10] N. Nogueira and Z. Cohn, "Trypanosoma cruzi: mechanism of entry and intracellular fate in mammalian cells," The Journal of Experimental Medicine, vol. 143, pp. 1402-1420, 1976.

[11] M. M. Piras, R. Piras, D. Henriquez, and S. Negri, "Changes in morphology and infectivity of cell culture-derived trypomastigotes of Trypanosoma cruzi," Molecular and Biochemical Parasitology, vol. 6, pp. 67-81, 1982.

[12] R. D. Ribeiro, R. A. Lopes, T. A. Garcia, and A. A. Carraro, "A new aspect of the morphological transformation of Trypanosoma cruzi brought about by environmental variation," Angewandte Parasitologie, vol. 31, no. 4, pp. 207-210, 1990.

[13] P. M. Schettino, S. Majumder, and F. Kierszenbaum, "Regulatory effect of the level of free $\mathrm{Ca}^{2+}$ of the host cell on the capacity of Trypanosoma cruzi to invade and multiply intracellularly," The Journal of Parasitology, vol. 81, pp. 597602, 1995.

[14] A. Zaidenberg, H. Tournier, G. Schinella, and H. Buschiazzo, "Trypanosoma cruzi: influence of human plasma on the morphogenesis of blood trypomastigotes in a cell-free culture media," Revista Latinoamericana de Microbiologia, vol. 37, pp. 71-77, 1995.

[15] E. Zweygarth, I. D. Gumm, M. A. Gray, J. K. Cheruiyot, P. Webster, and R. Kaminsky, "In vitro development of metacyclic Trypanosoma simiae derived from bloodstream trypomastigotes," Acta Tropica, vol. 46, pp. 277-282, 1989. 
[16] R. Manning-Cela, A. Cortes, E. Gonzalez-Rey, W. C. Van Voorhis, J. Swindle, and A. Gonzalez, "LYT1 protein is required for efficient in vitro infection by Trypanosoma cruzi," Infection and Immunity, vol. 69, pp. 3916-3923, 2001.

[17] W. De Souza, "Basic cell biology of Trypanosoma cruzi," Current Pharmaceutical Design, vol. 8, pp. 269-285, 2002.

[18] S. C. Bourguignon, C. B. Mello, D. O. Santos, M. S. Gonzalez, and T. Souto-Padron, "Biological aspects of the Trypanosoma cruzi (Dm28c clone) intermediate form, between epimastigote and trypomastigote, obtained in modified liver infusion tryptose (LIT) medium," Acta Tropica, vol. 98, pp. 103-109, 2006.

[19] V. T. Contreras, J. M. Salles, N. Thomas, C. M. Morel, and S. Goldenberg, "In vitro differentiation of Trypanosoma cruzi under chemically defined conditions," Molecular and Biochemical Parasitology, vol. 16, pp. 315-327, 1985.

[20] A. H. Kollien and G. A. Schaub, "The development of Trypanosoma cruzi in triatominae," Parasitology Today, vol. 16, no. 9, pp. 381-387, 2000.

[21] R. A. Mortara, L. M. Minelli, F. Vandekerckhove, V. Nussenzweig, and F. J. Ramalho-Pinto, "Phosphatidylinositol-specific phospholipase C (PI-PLC) cleavage of GPI-anchored surface molecules of Trypanosoma cruzi triggers in vitro morphological reorganization of trypomastigotes," The Journal of Eukaryotic Microbiology, vol. 48, no. 1, pp. 27-37, 2001.

[22] M. J. Soares, T. Souto-Padron, M. C. Bonaldo, S. Goldenberg, and W. De Souza, "A stereological study of the differentiation process in Trypanosoma cruzi," Parasitology Research, vol. 75, no. 7, pp. 522-527, 1989.

[23] Z. Brener, "Biology of Trypanosoma cruzi," Annual Review of Microbiology, vol. 27, pp. 347-382, 1973.

[24] H. Meyer, M. De Oliveira Musacchio, and I. De Andrade Mendonca, "Electron microscopic study of Trypanosoma cruzi in thin sections of infected tissue cultures and of blood-agar forms," Parasitology, vol. 48, pp. 1-8, 1958.

[25] D. B. Budzko, M. C. Pizzimenti, and F. Kierszenbaum, "Effects of complement depletion in experimental chagas disease: immune lysis of virulent blood forms of Trypanosoma cruzi," Infection and Immunity, vol. 11, no. 1, pp. 86-91, 1975.

[26] I. Mota and L. F. Umekita, "The effect of C3 depletion on the clearance of Trypanosoma cruzi induced by IgG antibodies," Immunology Letters, vol. 21, no. 3, pp. 223-226, 1989.

[27] N. Nogueira, C. Bianco, and Z. Cohn, "Studies on the selective lysis and purification of Trypanosoma cruzi," The Journal of Experimental Medicine, vol. 142, pp. 224-229, 1975.

[28] V. Ferreira, C. Valck, G. Sanchez, et al., "The classical activation pathway of the human complement system is specifically inhibited by calreticulin from Trypanosoma cruzi," The Journal of Immunology, vol. 172, pp. 3042-3050, 2004.

[29] K. Iida, M. B. Whitlow, and V. Nussenzweig, "Amastigotes of Trypanosoma cruzi escape destruction by the terminal complement components," The Journal of Experimental Medicine, vol. 169, pp. 881-891, 1989.

[30] K. A. Norris, B. Bradt, N. R. Cooper, and M. So, "Characterization of a Trypanosoma cruzi C3 binding protein with functional and genetic similarities to the human complement regulatory protein, decay-accelerating factor," The Journal of Immunology, vol. 147, no. 7, pp. 2240-2247, 1991. 

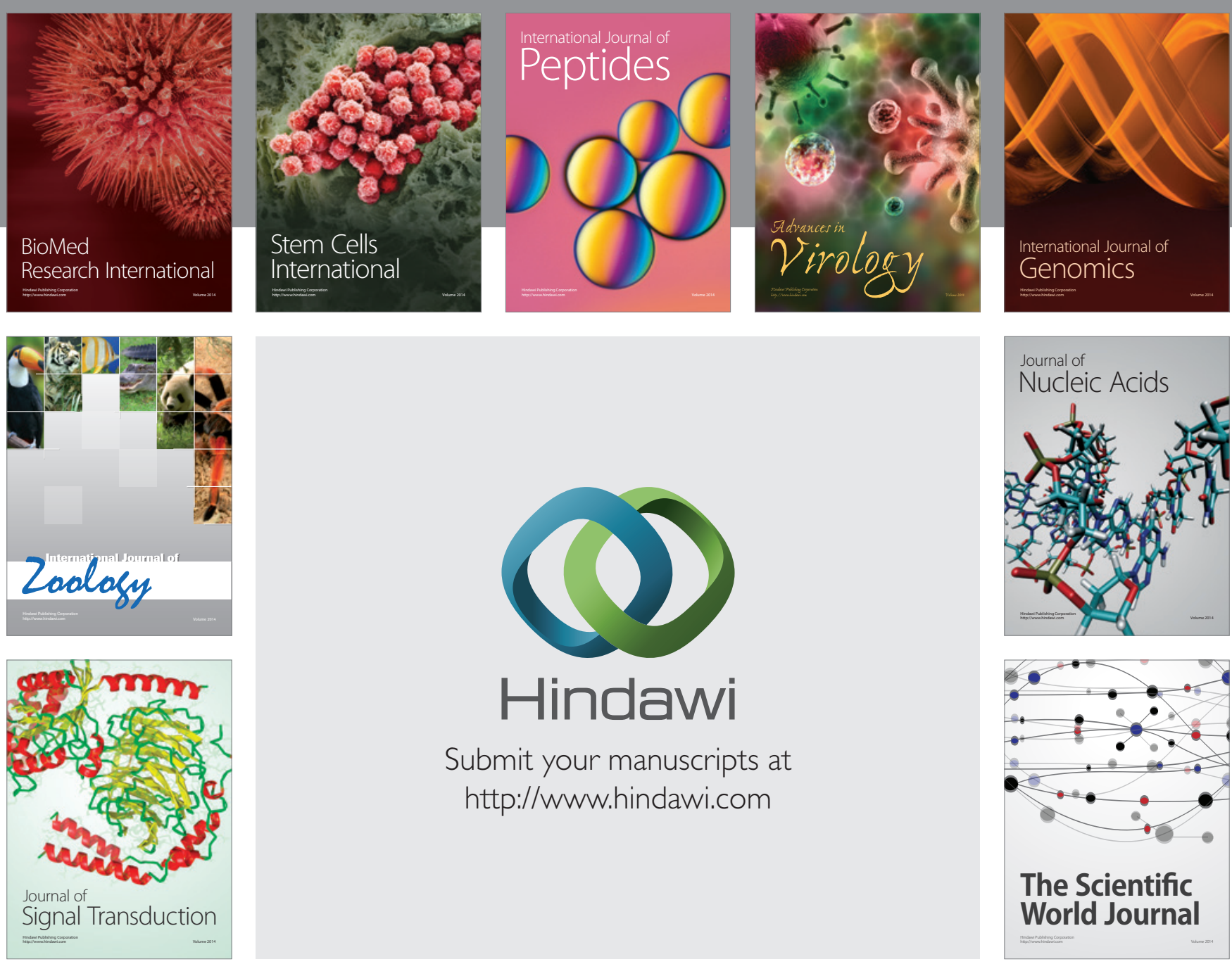

Submit your manuscripts at

http://www.hindawi.com
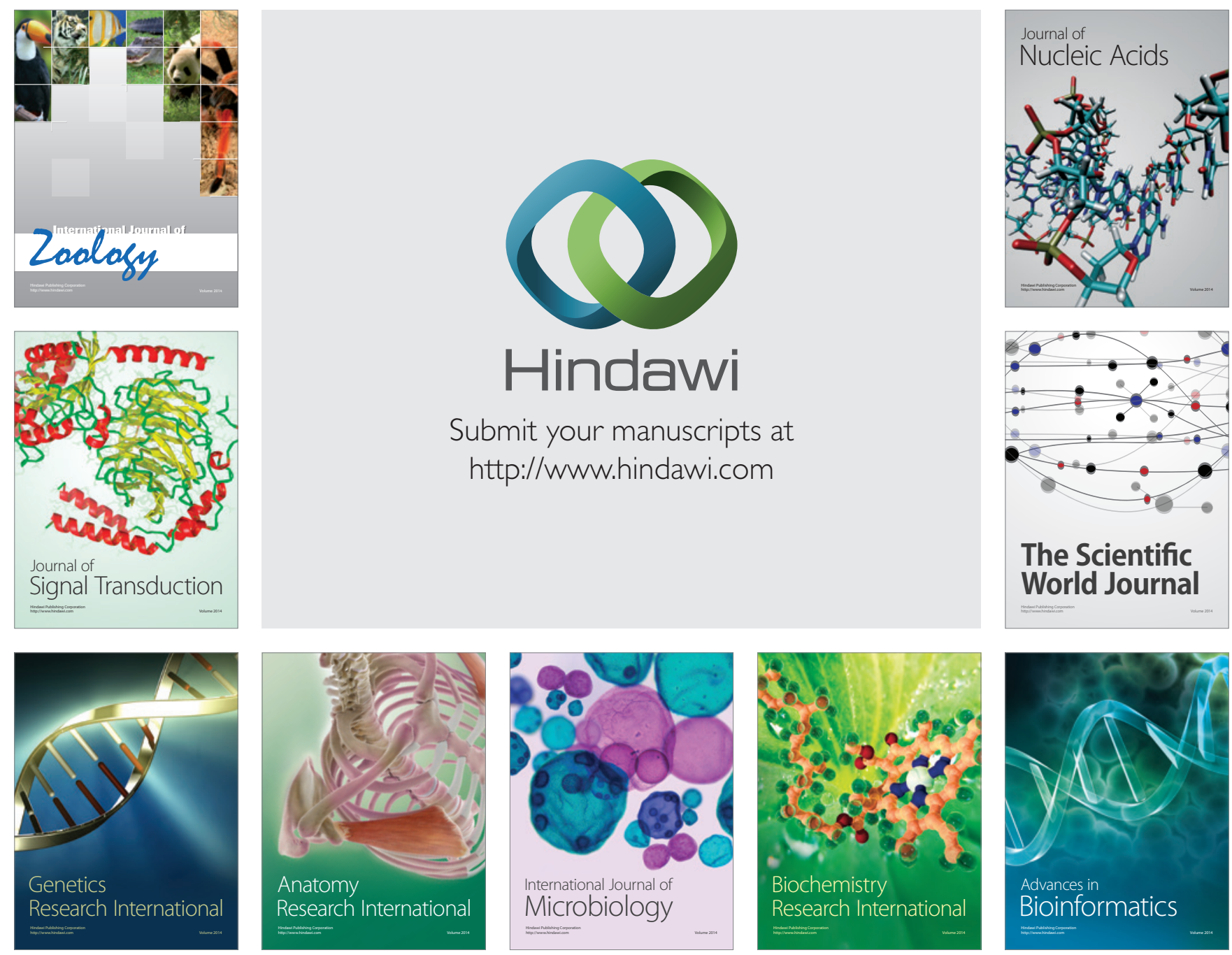

The Scientific World Journal
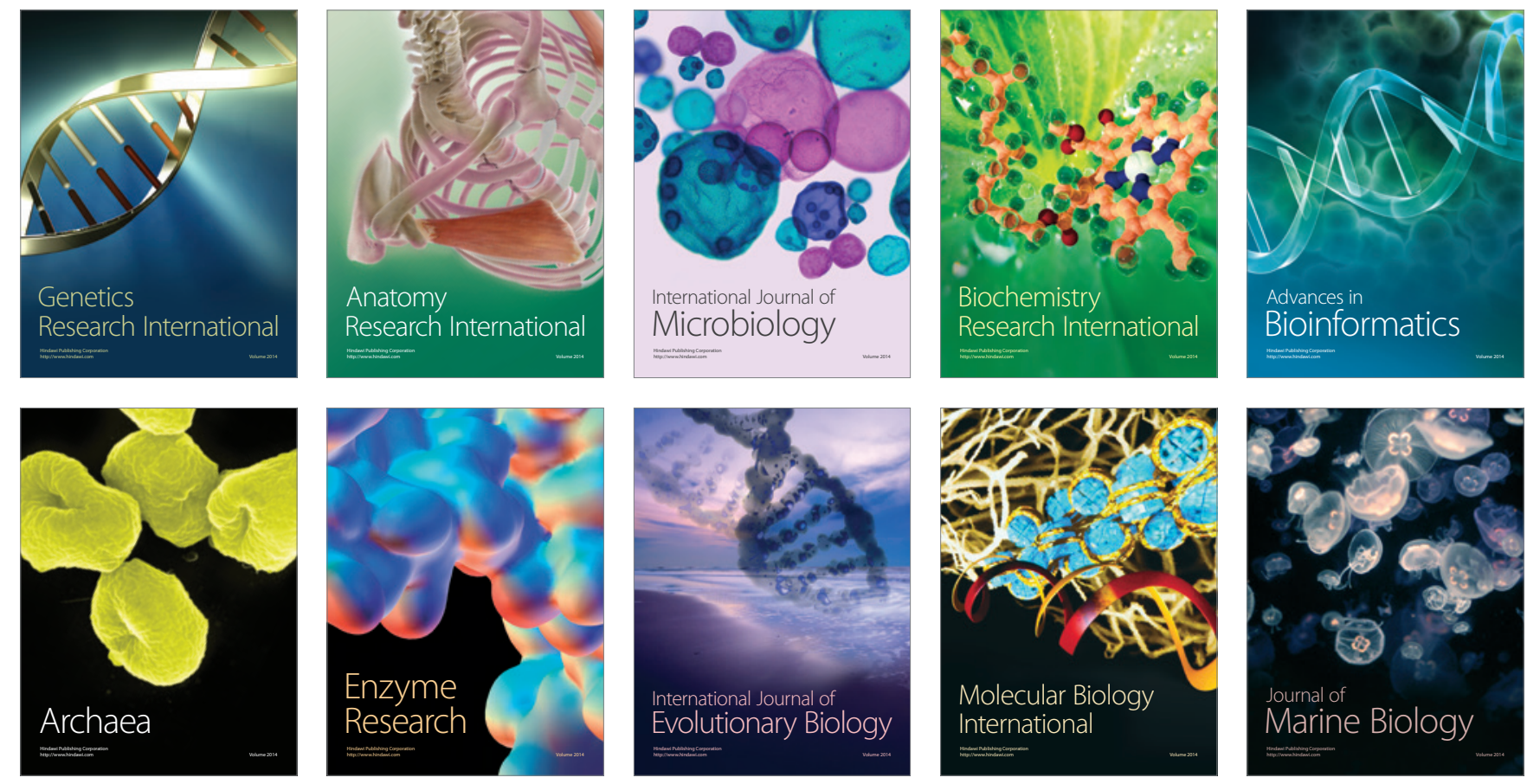